\title{
Comprehensive Analysis of Eph-Ephrin as Novel DLBC Biomarkers for Molecular Subtyping and the Predictability in Prognosis and Drug Response
}

Xiaoxi Li ( $\sim$ lixiaoxi@ujs.edu.cn )

Jiangsu University https://orcid.org/0000-0001-5399-5628

Chenxiao Zhang

Jiangsu University

Minyao Deng

Jiangsu University

Hui Qian

Jiangsu University

Primary research

Keywords: DLBCL, Chemoresistance, Eph Receptors, Ephrin Ligands, Expr-Ab

Posted Date: May 5th, 2021

DOI: https://doi.org/10.21203/rs.3.rs-452222/v1

License: @ (i) This work is licensed under a Creative Commons Attribution 4.0 International License.

Read Full License 


\section{Abstract}

Background: Receptor tyrosine kinases (RTKs) are key signal molecules for sustaining proliferative signaling and abnormal of RTKs appears in many cancers, including Lymphoid Neoplasm Diffuse Large B-cell Lymphoma (DLBC).

Methods: Cluster analysis based on RNA-seq and RPPA data were calculated to establish novel signature for molecular subtyping of DLBC. Principal component analysis (PCA) was used to evaluation relationship of selected genes.

Results: EPHB4, EPHB6, EFNA3, EFNA4, EFNB1 are 5 specific genes in DLBC and their expression level are significantly relevant to poor prognosis. Integration analysis of DLBC Expr-Ab signature discovered five DLBC related Eph-Ephrin genes may be involved in epigenetic regulation in DLBC progress. Four novel clusters based on Expr-Ab are generated and we first link five Eph-Ephrin to well-defined oncogenes as following: EPHB4-BCL6; EFNA3/EFNA4-MYC; EPHB6-EZH2; EFNB1-Epigenetic modulators. Drug response data involving 13 traditional and targeting drugs

Conclusions: Expr-Ab signature we established in this study indicates the power of both RNA-seq and RPPA data in developing and evaluating precision regimens. We also highlight Eph-Ephrin, as surface proteins, are powerful potentially biomarkers. Our finding underlines Eph-Ephrin as biomarkers for predicting prognosis and precision regimen for patients with lymphoma.

\section{Background}

Eph receptors are the largest family of receptor tyrosine kinases (RTKs). The role of Eph receptors and their Ephrin ligands in tumorigenesis and progression has been reported in many types of cancer[1-3]. Two characters make Eph-Ephrin proteins popular drug targets for novel anti-tumor therapy. One is that Eph-Ephrin proteins are localized on cell surface. Both Eph receptors and their Ephrin ligands including two types termed as A and B. A-type Eph, B-type Eph and B-type Ephrin are single transmembrane proteins and A-type Ephrin A is glycosyl phosphatidylinositol (GPI)-linked cell surface-bound proteins. Due to their roles in promoting proliferation of cancer cells, Eph-Ephrin proteins have become popular targets by antibody-based therapy. The other is that both Eph and Ephrin proteins are tyrosine kinases, which make them druggable targets by small molecular inhibitors. Hence, a tremendous amount of work, especially on antibody-based drugs, is carried out and developed based on various strategies $[4,5]$. However, Eph-Ephrin is a large family which is composed of 22 members. The expression pattern of 22 Eph-Ephrin members in various types of normal tissue and cancer are very complicated to understand. The Lacking of comprehensive analysis of Eph-Ephrin has become a stumbling block in research and drug development.

Multi-omics technologies, especially sequencing and protein array, have rapidly developed in recent years. These technologies allow comprehensive analysis of diseases at an individual level at low costs. One of the most affected diseases by multi-omics technologies is cancer. Precision cancer medicine involves in 
all aspects of cancer treatment, one of which is precision individual regiments. Genetic testing for therapy with targeting drugs, known as Companion Diagnostics (CDs), based on sanger sequencing, next generation sequencing (NGS) and quantitative PCR, are one applied area in clinical. While, genetic testing can just provide information of individual genetic variation, instead of predicting drug response in patients. Hence, valuable information with clinical impacts should be further mined. Lots of international projects aiming at precision cancer medicine have been proposed and finished in the past ten years. The projects include NGS based TCGA[6] (The Cancer Genome Atlas), cell line based CCEL[7] (Cancer Cell Lines Encyclopedia), cell viability measure based GDSC[8] (Genomics of Drug Sensitivity in Cancer), RPPA (Revers Phase Protein microArray) based TCPA[9] (The Cancer Proteome Atlas) and so on.

Lymphoma and leukemia are aggressive blood cancer involving all age groups. One subtype of lymphoma, diffused large B cell lymphoma (DLBCL), is the largest one in Non-Hodgkin Lymphoma (NHL) and has poor prognosis for nearly $40 \%$ of patients[10]. A high proportion of patients with poor prognosis will suffer central neurol system (CNS) involvement. Chemotherapeutic drugs can't reach lymphoma cells at effective concentration because of the Blood-Brain-Barrier (BBB), which is one major reason that patients can't benefit from chemotherapy. Although abnormal genetic variation caused by deletion, mutation, amplification involving B cell receptor signaling pathway, NF-KB signaling pathway are discovered by whole exons sequencing and other technologies, biomarkers for predicting prognosis and chemoresistance are still extremely deficient[11].

In this study, we identified major Eph-Ephrin genes in Lymphoid Neoplasm Diffuse Large B-cell Lymphoma (DLBC). DLBC is defined by TCGA, which contains DLBCL, Burkitt lymphoma and others. Next, we integrate RNA-seq data of Eph-Ephrin and DLBCL signature genes and RPPA data of DLBCL signature proteins (termed as 'Expr-Ab') to discover Eph-Ephrin pattern in DLBC and predict their possible function by clustering characteristics. Last, we explore the predictability of Expr-Ab on drug action based on available GDSC data, from which lots of Drug-Expr-Ab interaction can be further mined. We expect integrative strategies as Drug-Expr-Ab and beyond can be fully exploited in various cancer omics data to meet demand for diagnosis and therapeutics.

\section{Method}

Expression Data Retrieval and Analysis

Expression data (RESM,20180929) and RPPA data (20181003) of cell lines analyzed in this paper were originally downloaded from CCLE database (https://portals.broadinstitute.org/ccle). RPPA data of 33 DLBC patients (TCGA-DLBC-L4) was downloaded from TCPA database (https://tcpaportal.org/tcpa). IC50 data of 13 indicated drugs were downloaded from GDSC database (https://www.cancerrxgene.org/). Survival analysis of Eph-Ephrin genes was conducted using the SurvExpress online tool (http://bioinformatica.mty.itesm.mx:8080/Biomatec/SurvivaX.jsp). Microarray expression data of Lenz Staudt Lymphoma (GSE10846) contains detailed available clinical data of 420 DLBC patients. The 
optimized Maximize algorithm that attributes a minimal p-value to a risk group was performed and hazard ratio $(95 \%$ confidence interval $(\mathrm{Cl}))$ were processed using $\mathrm{R}$ studio.

All RNA-seq data and RPPA data were normalized to weed out the differences. Clustered heatmap and the correlation coefficients for were calculated and produced in R studio. Principal component analysis (PCA) was performed using the FactoMineR package in $\mathrm{R}$ studio.

\section{Results}

\section{EFNBA3, EFNA4, EFNB1, EPHB4, EPHB6 are identified as major expressed genes in DLBC}

To evaluate the expression pattern of Eph-Ephrin, we first analyse RNA-seq data of all 22 Eph-Ephrin members in 130 leukemia and lymphoma cell lines from the CCLE database. We find EPHB4, EFNBA3, EFNA4, EFNB1 are genes high expressed in most cell lines. EPHB1, EPHA1 and others are found highly expressed in specific cell lines (Supplementary Fig. 1A). Moreover, EPHB4, EPHB6, EPHA1 are highly expressed in ALL/AML cell lines, while EPHB1 are highly expressed in a small group of uncertain classified cell lines. Then, we performed the same analysis in 39 DLBC cell lines. EFNBA3, EFNA4, EFNB1, EPHB4, EPHB6 are identified as major expressed genes in DLBC (Fig. 1A). Further, we select 10 widely used cell lines for expression pattern and PCA analysis, in which NAMALWA, SU-DHL-4, SU-DHL-6, SUDHL-10, RAJI, DAUDI are classified in DLBC and THP-1, U-937, JURKAT, HL60 are classified in non-DLBC. We found EFNB1/EPHB6 and EFNBA3/EFNA4/EPHB4 are two significant differential expressed groups. The EFNBA3/EFNA4/EPHB4 group tends to be high expressed in DLBC cell lines, while the EFNB1/EPHB6/EPHA1 group are high expressed in non-DLBC cell lines (Fig. 1B). Further PCA analysis shows that EFNBA3/EFNA4/EPHB4 and EFNB1/EPHB6/EPHA1 are identified as two independent gene groups based on expression pattern.

Next, to determine the predictability of Eph-Ephrin members in prognosis, we analyzed microarray expression data of Lenz Staudt Lymphoma (GSE10846), which contains detailed available clinical data of 420 DLBC patients. Hazard Ratio and $p$ value of 22 Eph-Ephrin members show most Eph-Ephrin members are significantly relative to patients' prognosis (Supplementary Fig. 1B). Further, we focus on EFNBA3, EFNA4, EFNB1, EPHB4, EPHB6 according to their high expression in DLBC. Further, the combination of the 5 Eph-Ephrin genes (termed as 'combined-5') into over survival analysis shows more than 10 fold change in $p$ value, which means the combined- 5 has better performance in risk grouping than anyone of the 5 genes (Fig. 1D-E). From expression data we found low expression of EFNA3/EFNA4 are enriched in high-risk group with poor prognosis, in which EFNB1/EPHB4/EPHB6 are higher expression than low-risk group (Fig. 1F). These results indicate the expression data of EFNBA3/EFNA4/EFNB1/EPHB4/EPHB6 at mRNA level has the potential as diagnostic biomarkers to predict DLBC patients' prognosis.

Integrative analysis of Eph-Ephrin and DLBC abnormal pathway by mining multi-omics data 
To predict functions of Eph-Ephrin genes in lymphomas, we integrate two types of expression data involving DLBC major abnormal genes/pathway. One is mRNA expression data obtained by RNA-seq, and the other is protein expression data obtained by RPPA. By integrating expression data of Eph-Ephrin genes, we expect to predict which DLBC pathway the Eph-Ephrin genes are involved in. Firstly, we analyze RNA-seq data of one DLBC signature gene-set and the five blood-specific Eph-Ephrin genes, involving 22 genes (termed as Eph-Ephrin gene network). Expression pattern of Eph-Ephrin gene network generates 4 clusters as following: cluster 1 is TP53/CDKN1A, cluster 2 is Eph-Ephrin involving groups in DLBC, cluster 3 is BCL6/CARD11/CD79A/CD79B, cluster 4 is BCL2/CD80/TNFAIP3 (Fig. 2A). As expected, all EphEphrin genes are clustered in one group. 3 signatures on the basis of the expression pattern are characterized in 39 DLBC cell lines. High expression of cluster 2 and low expression of cluster 3 are characteristics of signature 1. High expression of cluster 4 are characteristic of signature 2. Medium expression of cluster 3 are characteristic of signature 3. Surprisingly, the other genes in the Eph-Ephrin group are transcriptional regulators (e.g. MYC) and epigenetic modulators (e.g.

KMT2D/CREBBP/EP300/EZH2). Further correlation analysis shows independence of both the DLBC signature genes and Eph-Ephrin genes (Supplementary Fig. 2A).

To confirm whether analysis results produced by RNA-seq data will be in line with protein data by RPPA/antibody (term as 'Ab'). We further screen and set one DBLC signature by Ab panel, which consists of $10 \mathrm{Ab}$ panel filtered from $47 \mathrm{Ab}$. First, we select $47 \mathrm{Ab}$ that involving major DLBC pathway which are ErbB signaling pathway, B cell receptor signaling pathway and PI3K-Akt signaling pathway (Supplementary Table 1). Second, RPPA data of $47 \mathrm{Ab}$ in blood cancer are analyzed by clustering, correlation coefficient, PCA (Supplementary Fig. 3A-B). Lastly, 10 independent Ab are selected as Ab panel of DBLC signature, analysis is performed both in blood cancer (Supplementary Fig. 3C-E) and DLBC (Fig. 3A-C). Furthermore, another RPPA data of 33 patients from the TCPA project is analyzed using the same $10 \mathrm{Ab}$ data. We got similar correlation coefficient and PCA results generated from protein/RPPA data (Fig. 3D-F). Hence, the 10 DBLC signature of protein/RPPA data are well-established.

Next, we further integrate RNA-seq and RPPA data (term as 'Expr-Ab') to analyse Eph-Ephrin pattern, attempting to predict Eph-Ephrin function more accurately and reliably. Similar to the Eph-Ephrin gene network signature, 4 novel clusters based on Expr-Ab are generated as follows: Ab data PKCa and its phosphorylation at $\mathrm{S} 657$ are clustered into the BCL6/CARD11/CD79A/CD79B group; BCL2/CD80/TNFAIP3 in Eph-Ephrin gene network signature are divided into two groups in Expr-Ab signature. One is CD80/TNFAIP3 with Ab data phosphorylation of B-Raf at S445 and PI3K-p85. The other is a novel group consisting of Ab data EGFR/HER2/Bcl-2 and BCL2's RNA-seq data. The last group is made up of the Eph-Ephrin genes, DNA Damage Response (DDR) genes (ATM/TP53/CDKN1A), Epigenetic modulator genes and others (Fig. 4A). 4 signatures on the basis of the expression pattern are characterized. Low expression of cluster 1 and high expression of cluster 4 are characteristic of signature 1. High expression of cluster 3 are characteristic of signature 2. Medium expression of cluster 1 are characteristic of signature 3 . Medium expression of cluster $2 / 3$ are characteristic of signature 4 . Next, we analyse the expression pattern of Eph-Ephrin genes in DLBC Expr-Ab signature. We found Eph-Ephrin 
genes closely correlated with epigenetic modulators which are highly mutated genes in DLBC uncovered by whole-exon sequencing studies (Fig. 4B-C).

\section{Drug Response Prediction by Expr-Ab signature involving Eph-Ephrin.}

To evaluate prospects of Expr-Ab signature in clinical, drug response data from GDSC project are integrated to Expr-Ab signature analysis. Available Expr-Ab data and intact drug response data of 13 traditional/targeting drugs in 12 lymphoma cell lines are retrieved into analysis. The results show drug response pattern can be, to some extent, clustered and 5 specific Expr-Ab clusters are generated termed as 'PI3K pathway', 'BCL6', 'MYC', 'Epigenetic Modulators', 'BCL2-HER2' (Fig. 5A). The most remarkable Expr-Ab signature involves in high expression level of cluster 'BCL6', 'MYC', 'Epigenetic Modulators' in cell lines A3KAW and A4FUK, which are sensitive to most drugs. The most multiple resistant phenotype are enriched in high expression level of cluster 'BCL2-HER2' in cell lines GRANTA519 and KARPAS422.

For Eph-Ephrin genes, EPHB4 is clustering with BCL6, one feature gene in a subtype of DLBC; EFNA3/EFNA4 is clustering with MYC, another feature gene in a subtype of DLBC; EPHB6 is clustering with $E Z H 2$, which is a recent uncovered feature gene and a druggable drug targets; EFNB1 is clustering with epigenetic modulators including KMT2C/KMT2D/EP300/CREBBP, which underline the intrinsic connection of EFNB1 and epigenetic modulators. Targeting EFNB1 may be a shortcut to develop therapeutics treating epigenetic abnormal cancer.

\section{Discussion}

Before 2010, microarray was the main technology for high-throughput gene expression analysis[12]. The relative expression level of genes can be measured by hybridization of the probe on the chip with the nucleic acid of the sample. However, microarray technology can't measure the absolute value of gene expression. Therefore, the importance of genes with low or no expression may be overestimated, while the importance of genes with high expression may be ignored. After 2010, with the rapid development of next generation sequencing (NGS) technology, the cost of sequencing has been further reduced, and a large number of international systematic functional genome research projects have been implemented[13]. CCLE has carried out multi-omics analysis of more than 1000 cell lines involving dozens of cancer types established in the laboratory, which has improved the important resources for the study of gene function.

Eph-Ephrin contains 22 receptor-ligand family members. The Eph-Ephrin genes may have cell lineage specificity in the process of evolution, but Eph-Ephrin signaling pathway should be indispensable to all cell lineages. Therefore, each cell lineage must express several Eph-Ephrin genes. Although many members of Eph-Ephrin signaling pathway have been reported abnormal expressed in many cancers, systematic analysis of Eph-Ephrin in cancer and relationship of prognosis still lack. Here, we comprehensively analyse the expression pattern of Eph-Ephrin in DLBC on the basis of the CCLE database. Some of Eph-Ephrin genes, instead of all, must be indispensable for DLBC and gene expression is the basis of gene function. Hence, to find out key Eph-Ephrin genes in blood cancer or DLBC, we 
analyse RNA-seq data from CCLE. We suppose EPHB4 /EPHB6 are receptors and EFNA3/EFNA4/EFNB1 are ligands for most blood cancer or DLBC cell lines. Overall survival analysis indicates the five EphEphrin genes possess well prognostic predictability in DLBC. More comprehensive and deep analysis should be conducted on other types of blood cancer.

Integration of Eph-Ephrin genes with 17 DLBC signature genes generates 4 clusters as showed in Fig. 2 and all of the five Eph-Ephrin genes are clustered in one group. Destruction of B cell signaling pathway, such as low or no expression of CD79a and CD79b, and abnormal high expression of various transcription regulators, epigenetic modulators, Eph-Ephrin genes are the main feature of signature 1 cells. Similar results also appear in the Expr-Ab analysis in Fig. 4. It's a negative correlation between the expression of Eph-Ephrin and CD79a/CD79b in signature 1 cells, which indicates potential interaction between them according to the same subcellular localization.

Compared to signature 3, the feature of signature 2 in Eph-Ephrin gene network is high expression of cluster 4 (BCL2/CD80/TNFAIP3).

In the Expr-Ab analysis, the integration of expression data of RNA and protein can merge the advantages of RNA-seq and RPPA, to obtain more accurate classification of DLBC according to gene expression profile. The feature of signature 1 in the Expr-Ab is similar to signature 1 in Eph-Ephrin gene network. The feature of signature 2 in the Expr-Ab is high expression of cluster 3 (BCL2/EGFR/HER2), in which BCL2 is an anti-apoptosis gene and EGFR/HER2 are pro-proliferation genes. The feature of signature 3 and signature 4 in the Expr-Ab is expression pattern of cluster 1 (CARD11/CD79A/CD79B/BCL6/PKC-a). BCL6/PKC-a are high expression in signature 3 and low expression in signature 4. Hence, the Expr-Ab pattern is useful to classification of DLBC to the signature 1-4. For example, samples with high PKC-a (cluster 1) can be classified to the signature 3. Samples with high BCL2 (cluster 3 ) can be classified to the signature 2. Samples with high MYC (cluster 4) can be classified to the signature 1. Samples with low $\mathrm{PKC}-\mathrm{a} / \mathrm{BCL} 2 / \mathrm{MYC}$ can be classified to the signature 4.

After combining the drug response data from GDSC with the integrated Expr-Ab expression data, we found that similar drug response results can be clustered together based on the Expr-Ab. For example, A3KAW and A4FUK, which have similar expression pattern as the signature 1 of the Expr-Ab, have similar sensitive pattern to most of the 13 drugs. GRANTA519 and KARPAS422, which have similar expression pattern as the signature 2 of the Expr-Ab, have similar resistant pattern to most of the 13 drugs. These data show that the Expr-Ab expression data can reflect and predict the results of drug response. More detailed multi-omics expression data should be further integrated and analyzed to select a better gene set for predicting drug response.

In addition, it is surprising and interesting to link Eph-Ephrin genes to transcriptional regulators and epigenetic modulators. The Eph-Ephrin possess activity of kinase and may directly or indirectly regulate expression and active status of downstream targets through phosphorylation. Downstream targets of EPHB4, EPHB6, EFNA3, EFNA4, EFNB1 in DLBC should be identified in the future. 


\section{Conclusions}

In this study, we comprehensive analysis 22-gene expression pattern of Eph-Ephrin in leukemia and lymphoma from CCLE RNA-seq data and select five DLBC high-expression Eph-Ephrin genes, receptors EPHB4, EPHB6 and ligands EFNA3, EFNA4, EFNB1. Microarray expression data of Lenz Staudt Lymphoma GSE10846, including detailed available clinical data of 420 DLBC patients, are analysis to evaluation the performance of Eph-Ephrin genes in prognosis prediction. Overall survival data indicates expression level of the 5 Eph-Ephrin genes are significant related to risk grouping of patients.

Integration analysis of DLBC Expr-Ab signature discovered five DLBC related Eph-Ephrin genes may be involved in epigenetic regulation in DLBC progress. Four novel clusters based on Expr-Ab are generated. Cluster 1 is PKCa(Ab)/PKCa-S657(Ab)/BCL6/CARD11/CD79A/CD79B; Cluster 2 is CD80/TNFAIP3/B-RafS445(Ab)/PI3K-p85(Ab); Cluster 3 is EGFR(Ab)/HER2(Ab)/Bcl-2(Ab)/BCL2; Cluster 4 is EphEphrin/transcriptional factors/Epigenetic modulators.

Drug response prediction involving 13 traditional and targeting drugs by our Expr-Ab signature indicates the power of both RNA-seq and RPPA data in developing and evaluating precision regimens in clinical. At the same time, we highlight Eph-Ephrin are powerful potentially biomarkers to represent and replace other types of variations as following: EPHB4-BCL6; EFNA3/EFNA4-MYC; EPHB6-EZH2; EFNB1-Epigenetic modulators. Eph-Ephrin genes, other than non-cell surface proteins, have better potential to develop into molecular diagnostic targets. Our finding underlines Eph-Ephrin as biomarkers for predicting prognosis and precision regimen for patients with lymphoma.

\section{Declarations}

\section{Ethics approval and consent to participate}

Not applicable

\section{Consent for publication}

Not applicable

\section{Availability of data and materials}

All raw data are available from public database. $\mathrm{R}$ scripts used in this study are available from the corresponding author on reasonable request.

\section{Competing interests}

The authors declare that they have no competing interests

\section{Funding}


This research was supported by the National Natural Science Foundation of China (81900200), Natural Science Foundation of Jiangsu Province (BK20190840), State Key Laboratory of cell biology (SKLCB2018KF008).

\section{Authors' Contributions}

X.L. conceived the project and designed the analysis. C. Z., M. D., H. Q. discussed the results. X.L. wrote the manuscript. All authors reviewed and approved the final version of the manuscript.

\section{Acknowledgments}

We are grateful to Zhuang Wei for guidance on R studio and Yuji Shi for insightful advice.

\section{Abbreviations}

Receptor tyrosine kinases (RTKs)

Lymphoid Neoplasm Diffuse Large B-cell Lymphoma (DLBC)

diffused large B cell lymphoma (DLBCL)

Non-Hodgkin Lymphoma (NHL)

Principal component analysis (PCA)

RPPA (Revers Phase Protein microArray)

\section{References}

1. Kania A, Klein R. Mechanisms of ephrin-Eph signalling in development, physiology and disease. Nat Rev Mol Cell Biol [Internet]. Nature Publishing Group; 2016;17:240-56. Available from: http://dx.doi.org/10.1038/nrm.2015.16.

2. Pasquale EB. Eph-Ephrin Bidirectional Signaling in Physiology and Disease. Cell. 2008;133:38-52.

3. Pasquale EB. Eph receptors and ephrins in cancer: Bidirectional signalling and beyond. Nat Rev Cancer [Internet]. Nature Publishing Group; 2010;10:165-80. Available from: http://dx.doi.org/10.1038/nrc2806.

4. Barquilla A, Pasquale EB. Eph receptors and ephrins: Therapeutic opportunities. Annu Rev Pharmacol Toxicol. 2015;55:467-87.

5. Boyd AW, Bartlett PF, Lackmann M. Therapeutic targeting of EPH receptors and their ligands. Nat Rev Drug Discov [Internet]. Nature Publishing Group; 2014;13:39-62. Available from: http://dx.doi.org/10.1038/nrd4175.

6. Morin RD, Mendez-Lago M, Mungall AJ, Goya R, Mungall KL, Corbett RD, et al. Frequent mutation of histone-modifying genes in non-Hodgkin lymphoma. Nature. 2011. 
7. Ghandi M, Huang FW, Jané-Valbuena J, Kryukov GV, Lo CC, McDonald ER, et al. Next-generation characterization of the Cancer Cell Line Encyclopedia. Nature. 2019.

8. Garnett MJ, Edelman EJ, Heidorn SJ, Greenman CD, Dastur A, Lau KW, et al. Systematic identification of genomic markers of drug sensitivity in cancer cells. Nature. 2012.

9. Li J, Lu Y, Akbani R, Ju Z, Roebuck PL, Liu W, et al. TCPA: A resource for cancer functional proteomics data. Nat. Methods. 2013.

10. Wang L, Li L, Young KH. New agents and regimens for diffuse large B cell lymphoma. J. Hematol. Oncol. 2020.

11. Pasqualucci L, Dalla-Favera R. Genetics of diffuse large b-cell lymphoma. Blood. 2018.

12. Alizadeh AA, Alizadeh AA, Eisen MB, Eisen MB, Davis RE, Davis RE, et al. Distinct types of diffuse large B-cell lymphoma identified by gene expression profiling. Nature. 2000.

13. Reddy A, Zhang J, Davis NS, Moffitt AB, Love CL, Waldrop A, et al. Genetic and Functional Drivers of Diffuse Large B Cell Lymphoma. Cell. 2017.

\section{Figures}


Figure 1

A

Eph-Ephrin in DLBC
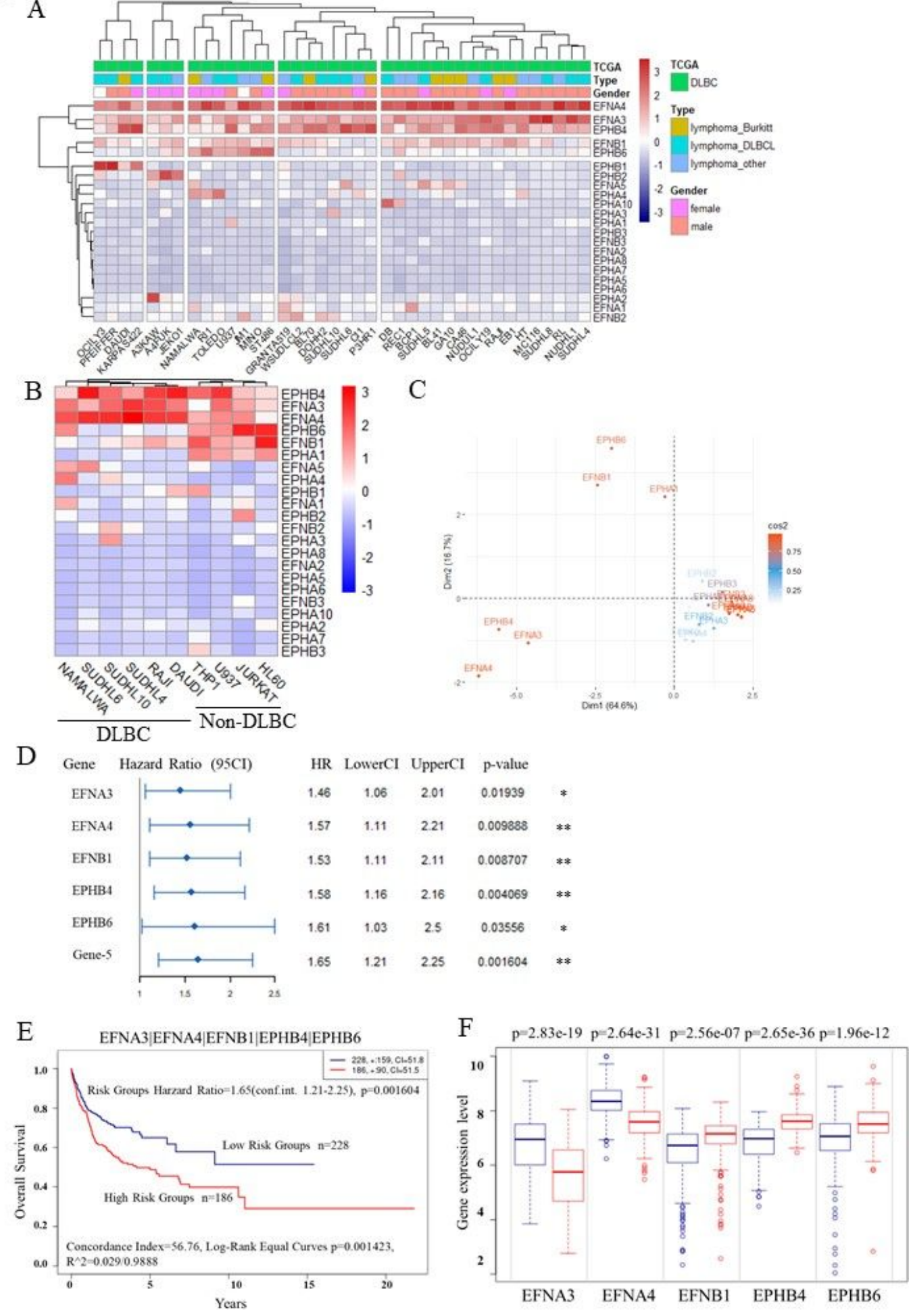

\section{Figure 1}

Expression pattern and prognostic potential of Eph-Ephrin genes in DLBC. (A) Relative expression of 22 Eph-Ephrin genes in 39 DLBC cell lines annotated by TCGA. (B) Relative expression of 22 Eph-Ephrin genes in 10 selected cell lines. NAMALWA, SU-DHL-4, SU-DHL-6, SU-DHL-10, RAJI, DAUDI are DLBC cell lines and THP-1, U-937, JURKAT, HL60 are non-DLBC cell lines. (C) Principal component analysis (PCA) of 22 Eph-Ephrin genes on the basis of expression pattern in 10 selected cell lines. (D) Hazard ratio (HR) 
calculated from high risk group and low risk group of GSE10846 determined by the SurvExpress optimized algorithm on the basis of individual or combined Eph-Ephrin expression. Combined "Gene-5" are EFNBA3, EFNA4, EFNB1, EPHB4, EPHB6. (E) Kaplan-Meier curves of high risk and low risk population on the basis of combined "Gene-5" expression level. Risking grouping was conducted through an optimization algorithm. $\left({ }^{*}, p<0.05 ; * \star, p<0.01\right)$. (F) The expression level of EFNBA3, EFNA4, EFNB1, EPHB4, EPHB6 in low risk group and high risk group.

\section{Figure 2}

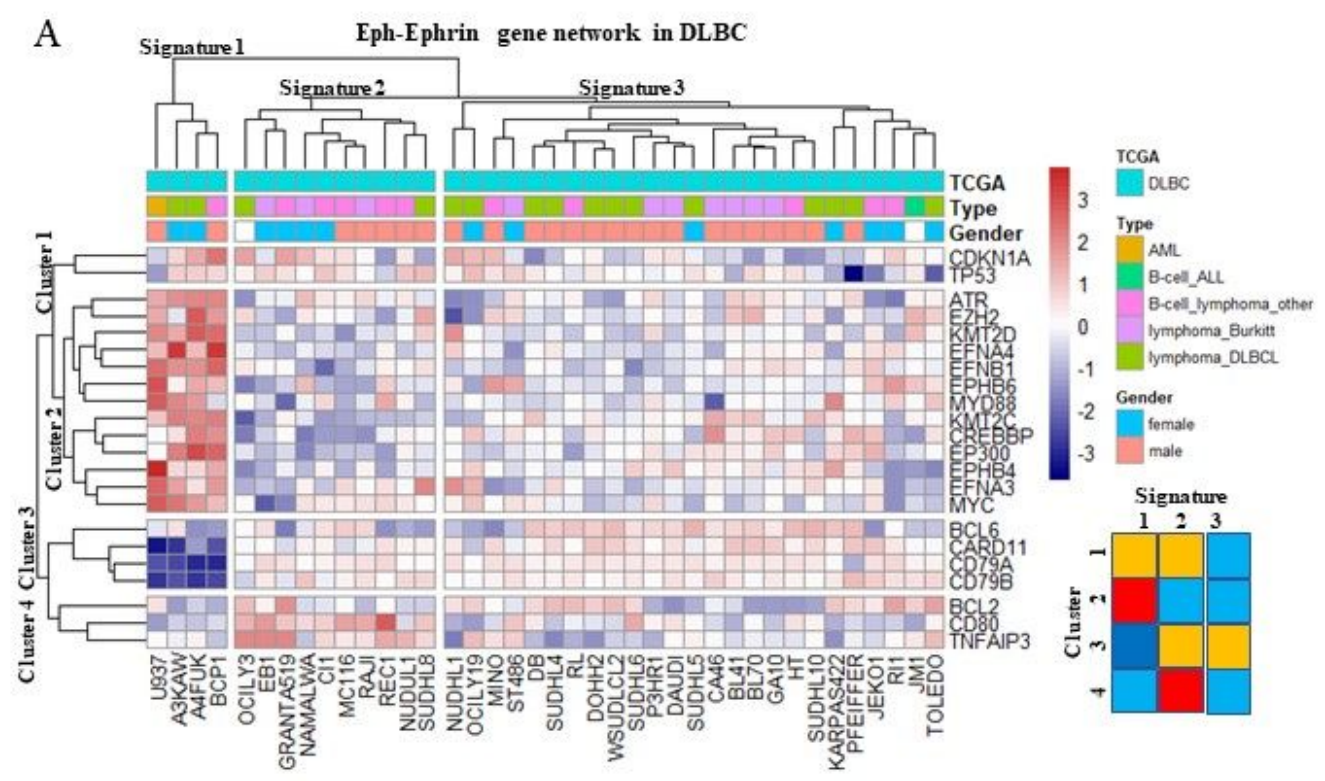


Expression pattern of Eph-Ephrin gene network in DLBC. (A) Expression pattern of 22 genes composed of one DLBC signature gene-set and EFNBA3, EFNA4, EFNB1, EPHB4, EPHB6 in DLBC cell lines. The 22 genes are termed as Eph-Ephrin gene network.

\section{Figure 3}

A Key Proteins in DLBC

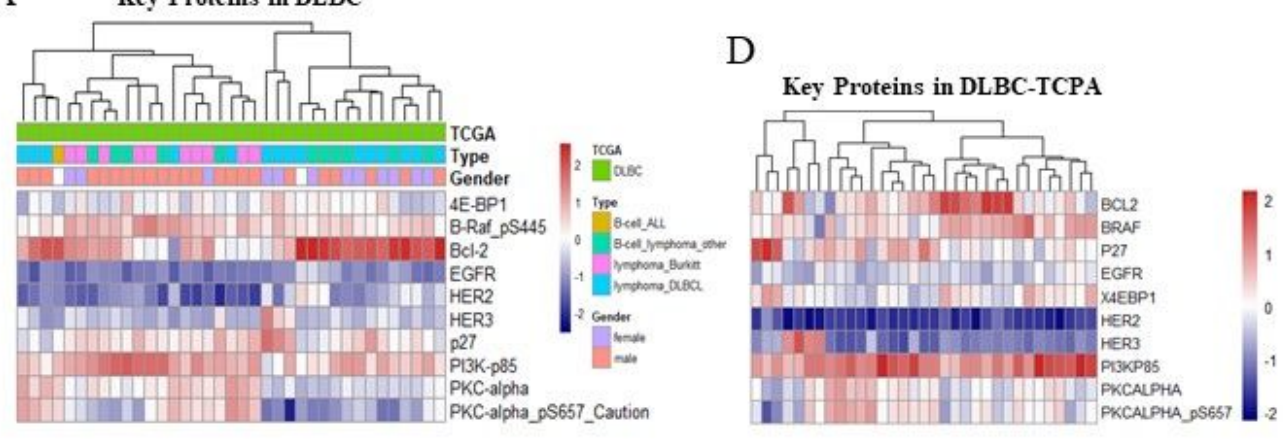

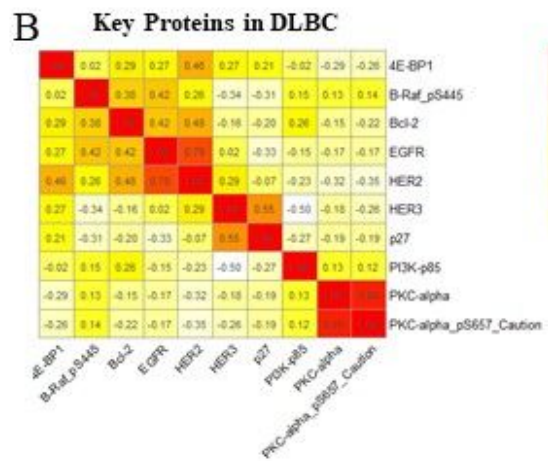

C Key Proteins in DLBC

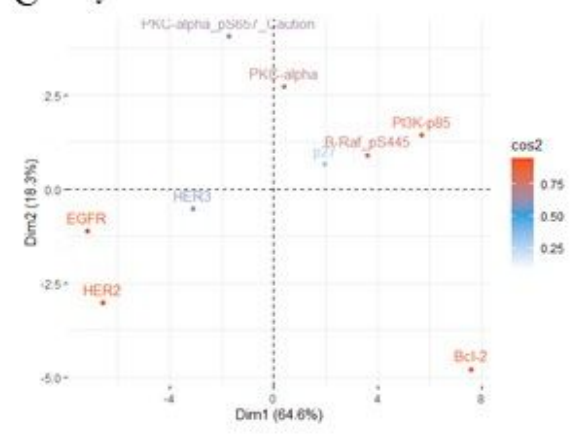

E Key Proteins in DLBC-TCPA

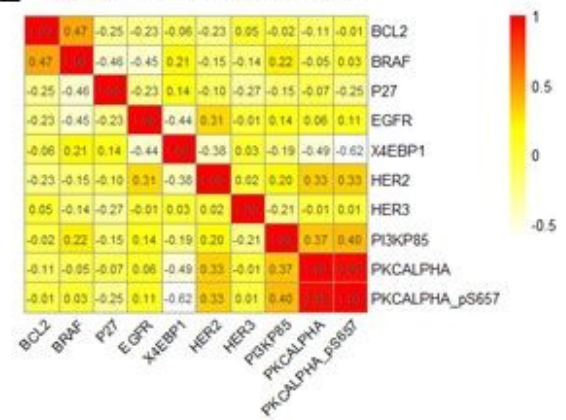

F Key Proteins in DLBC-TCPA

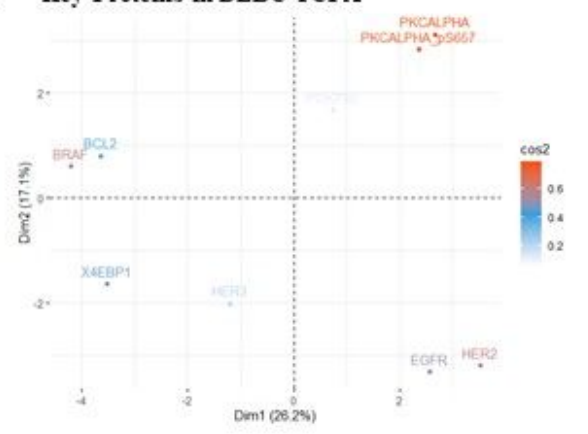

Figure 3

Expression pattern of key proteins in DLBC. (A) Expression pattern of 10 key proteins in 39 DLBC cell lines based on RPPA data. (B-C) The correlation coefficients and principal component analysis (PCA) of 10 key 
proteins on the basis of RPPA data in 39 DLBC cell lines. (D) Expression pattern of 10 key proteins in 33 DLBC TCPA samples based on RPPA data. (E-F) The correlation coefficients and principal component analysis (PCA) of 10 key proteins on the basis of RPPA data in 33 DLBC TCPA samples.

Figure 4
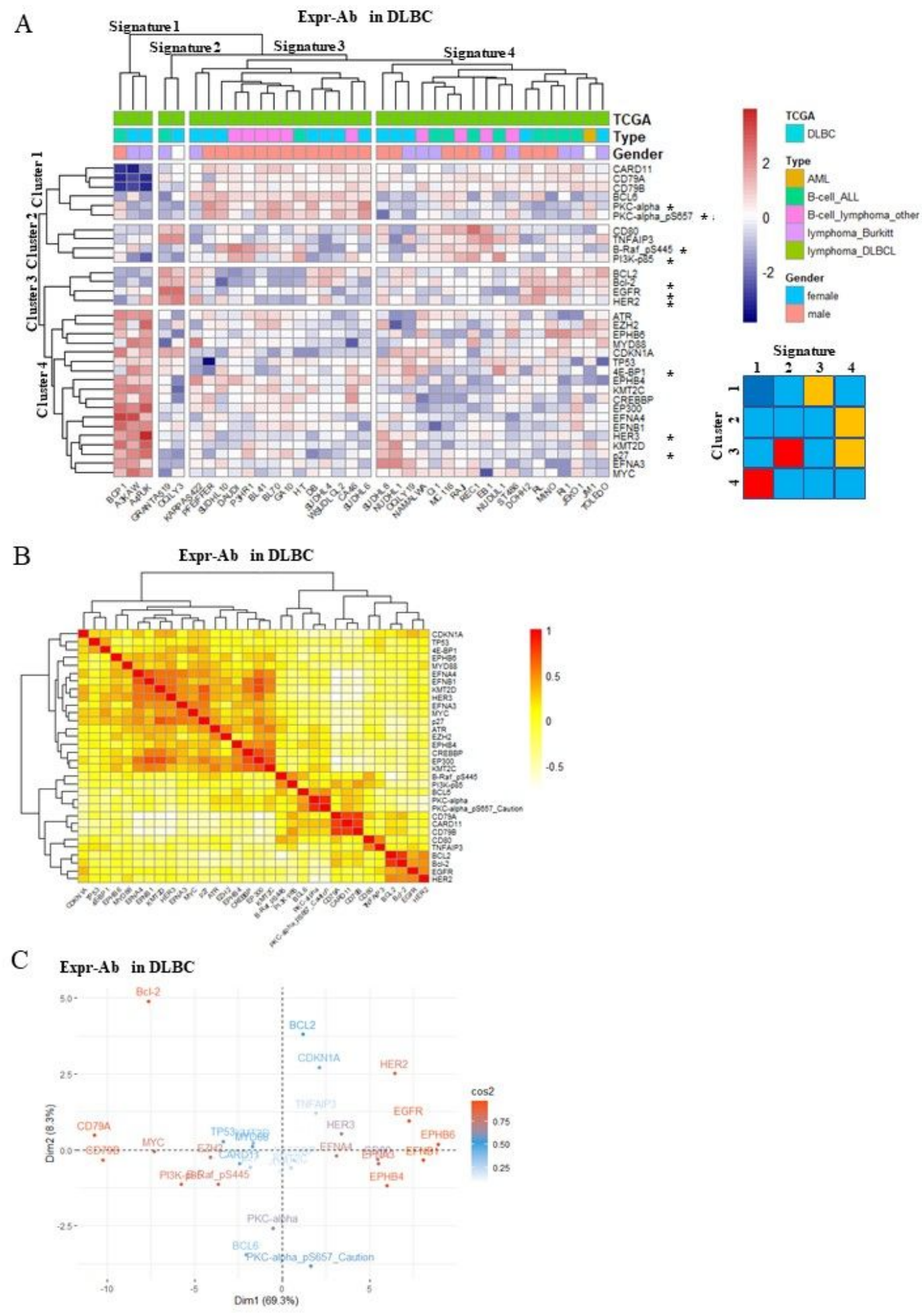

Figure 4

Integrative analysis of Expr-Ab multi-omics data in DLBC. (A) Expression pattern of Expr-Ab signature in DLBC. Expr-Ab signature includes RPPA data of 10 proteins and RNA-seq data of 22 genes. (B-C) The 
correlation coefficients and principal component analysis (PCA) of Expr-Ab signature in 39 DLBC cell lines.

\section{Figure 5}

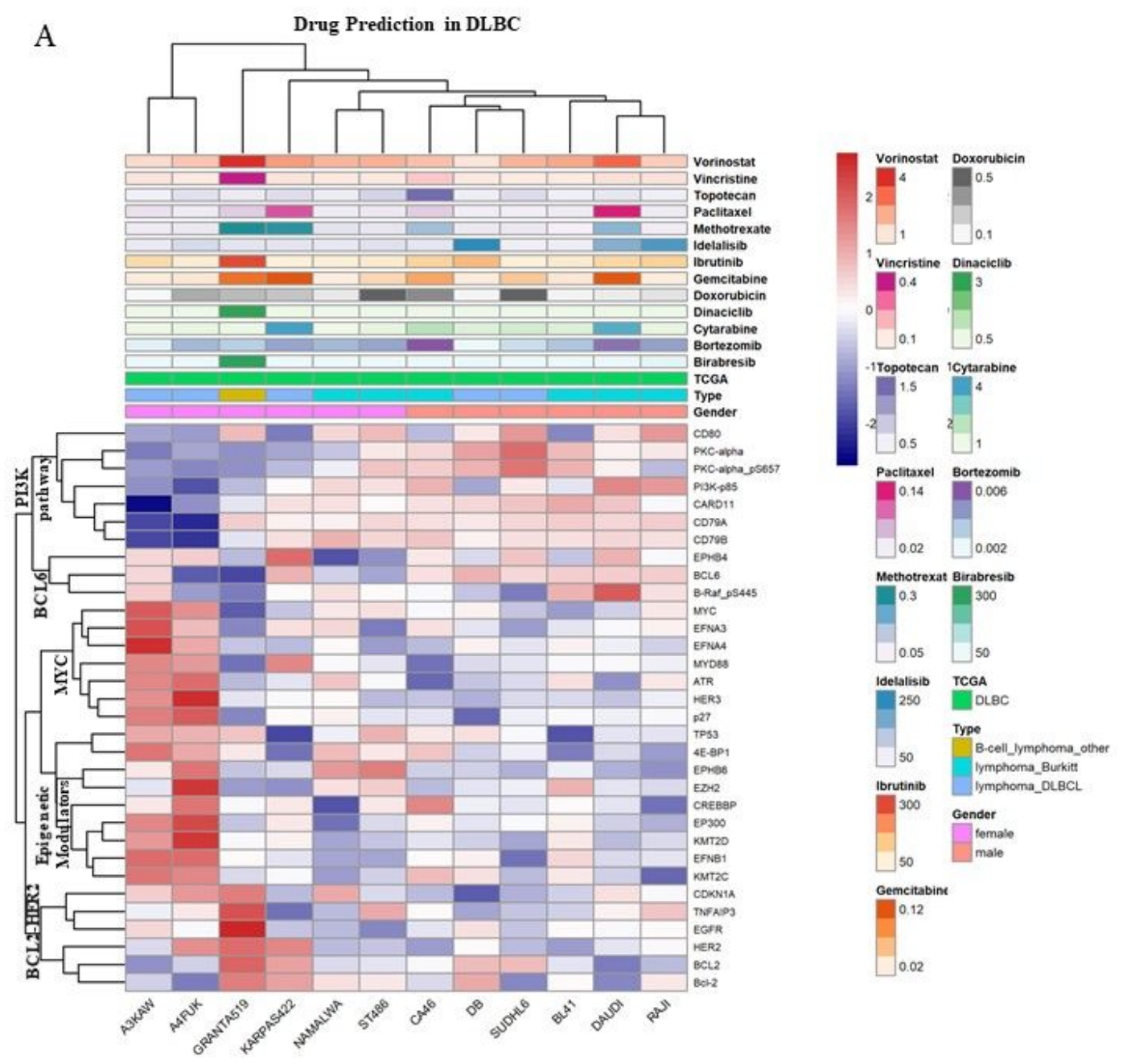

Figure 5

Analysis of the predictability between Expr-Ab signature and drug response. (A) Expr-Ab signature and paired drug response data in 12 blood cancer cell lines. The unit of drugs' IC50 is $\mu \mathrm{M}$. 


\section{Supplementary Files}

This is a list of supplementary files associated with this preprint. Click to download.

- SFigure1.jpg

- SFigure2.jpg

- SFigure3.jpg 